ESAIM: PROCEEDINGS AND SURVEYS, June 2018, Vol. 63, p. 44-59

L. Grigori, C. Japhet, P. Moireau, Editors

\title{
FEM-BEM COUPLING FOR ELECTROMAGNETISM WITH THE SPARSE CARDINAL SINE DECOMPOSITION ${ }^{*}, *$
}

\author{
Francois Alouges ${ }^{1}$, Matthieu Aussal ${ }^{2}$ and Emile Parolin ${ }^{3}$
}

\begin{abstract}
This paper presents a FEM-BEM coupling method suitable for the numerical simulation of the electromagnetic scattering of objects composed of dielectric materials and perfect electric conductors. The originality of the approach lies in part in the use of the newly proposed Sparse Cardinal Sine Decomposition (SCSD) method for the BEM part of the computation and the fact that the simulation software is almost entirely written in MATLAB.

The performance of the method is illustrated by the computation of the electromagnetic scattering by an UAV-like object with two RAM regions proposed in the workshop ISAE EM 2016.

Résumé. Cet article présente une méthode de couplage de type FEM-BEM adaptée à la simulation numérique des problèmes de diffraction électromagnétique d'objets composés de parties parfaitement conductrices et de matériaux diélectriques. L'originalié de l'approche réside, d'une part, dans l'utilisation de la nouvelle méthode décomposition creuse en sinus cardinal (SCSD) pour la partie BEM et, d'autre part, dans le fait que le programme de calcul est presqu'entièrement écrit en MATLAB.

La performance de la méthode est illustrée par le calcul de la diffraction électromagnétique d'un objet en forme d'UAV contenant deux régions absorbantes tel qu'il a été proposé au workshop ISAE EM 2016.
\end{abstract}

\section{INTRODUCTION}

It has by now become common to solve scattering electromagnetic problems in harmonic regime with the so-called Boundary Element Method (BEM). It permits, with quite a good precision, to represent the electromagnetic field in the unbounded region around the scattering object with a discretization only realized at the surface of the scattering object. This reduces a three-dimensional problem to a bi-dimensional one. However, with the development of such methods, the main difficulty that one encounters is that these formulations lead classically to linear systems that involve dense matrices (in contrast with volumic finite element methods, for instance). Several methods, among which the most famous are probably the FMM (Fast Multipole Method) (see e.g. $[9,16]$ ) or $\mathcal{H}$-matrices (see [11]), have been used to circumvent this difficulty. Those methods operate

* We gratefully thank Jérôme Simon from ONERA for having provided us with the UAV surface mesh, and Robin Gueguen for his help in order to build the $3 D$ mesh.

** This work was done with the support of the French Direction Générale de l'Armement.

${ }^{1}$ Centre de Mathématiques Appliquées, Ecole polytechnique et CNRS, UMR 7641, Route de Saclay, 91128 Palaiseau Cedex, université Paris-Saclay. francois.alouges@polytechnique.edu .

${ }^{2}$ Centre de Mathématiques Appliquées, Ecole polytechnique et CNRS, UMR 7641, Route de Saclay, 91128 Palaiseau Cedex, université Paris-Saclay. matthieu.aussal@polytechnique.edu .

${ }^{3}$ Centre de Mathématiques Appliquées, Ecole polytechnique et CNRS, UMR 7641, Route de Saclay, 91128 Palaiseau Cedex, université Paris-Saclay. emile.parolin@polytechnique.edu .

(C) EDP Sciences, SMAI 2018 
by compressing the representation of the underlying linear system. This enables to store an approximation of the matrix while speeding up the time of a matrix-vector multiplication.

More recently a new compression technique, the Sparse Cardinal Sine Decomposition (SCSD), was developed for the same goal $[1,4]$, and applied to point to point gravitational interactions or acoustic scattering [2]. A more recent application to the Stokes problem must also be pointed out [3].

The purpose of the paper is therefore twofold:

- On the one hand, we describe the application of the SCSD to the simulation of electromagnetic scattering problem using the BEM and more precisely the coupling of the method with the discretization of dielectric regions using the more classical finite element method.

- On the other hand we show the performances of a computation code that we have written in MATLAB, based on this method, on an industrial test case. In particular, we provide the user with the results obtained for the workshop ISAE EM 2016, where the electromagnetic scattering of a complex object, consisting both of a perfect electric conductor and dielectric regions, has to be computed.

This paper is organized as follows. Section 1 is devoted to the classical integral equation BEM framework for electromagnetic scattering and the coupling with a volumic finite element discretization for the dielectric materials. Section 2 explains the fundamentals of the SCSD, and eventually Section 3 provides the reader with the results on classical numerical tests for validation together with the test case coming from the workshop ISAE EM 2016.

\section{Problem Formulation}

The problem that we have in mind is the electromagnetic scattering in the three dimensional space $\mathbb{R}^{3}$ of an object which is partly a perfect electric conductor (PEC) and partly composed of a dielectric material. We suppose that the obstacle is lit by an incident electromagnetic plane wave $\left(E_{\text {inc }}, H_{\text {inc }}\right)$. The object generates an outgoing scattered wave $\left(E_{\mathrm{sc}}, H_{\mathrm{sc}}\right)$, and we call

$$
\left(E_{\mathrm{tot}}, H_{\mathrm{tot}}\right)=\left(E_{\mathrm{inc}}, H_{\mathrm{inc}}\right)+\left(E_{\mathrm{sc}}, H_{\mathrm{sc}}\right)
$$

the total electromagnetic field in the exterior domain, that we denote by $\Omega^{+}$. There exists also a transmitted electromagnetic field, which we call $\left(E_{\mathrm{tr}}, H_{\mathrm{tr}}\right)$, in the dielectric domain, denoted by $\Omega_{\mathrm{d}}^{-}$. Let $\Omega_{\mathrm{PEC}}^{-}$represent the PEC domain, we note $\Gamma_{\mathrm{PEC}}$ the interface vacuum-PEC, $\Gamma_{\mathrm{d}}$ the interface vacuum-dielectric, and $\Sigma$ the interface between the PEC and the dielectric domains. A sketch of the problem under consideration is given in Figure 1.

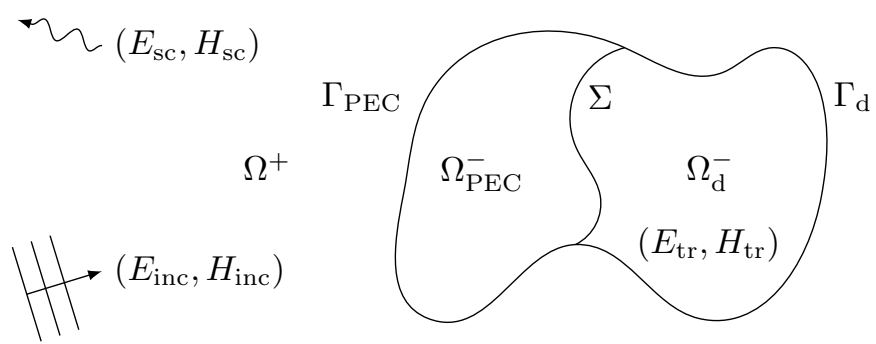

Figure 1. Sketch of the problem.

All fields satisfy the harmonic Maxwell equations with a wave number depending on the electric permittivity and magnetic permeability of the considered medium. In the following, we denote the wavenumber of the vacuum by $k$ and the wavenumber of the dielectric material by $k_{d}$. We also introduce $\epsilon_{r}$ and $\mu_{r}$ respectively the relative permittivity and the relative permeability of the dielectric material so that we have $k_{d}=\sqrt{\epsilon_{r} \mu_{r}} k$. 
In the sequel, we derive the equations that we solve in the last part of the paper. The vacuum is taken into account through an integral equation from the surfacic currents (electric and magnetic) at the surface of the object, while the dielectric part of the object is solved through a volumetric finite element method applied to Maxwell equations. The final coupling is obtained by using adequate transmission conditions, namely the continuity of the tangential traces of the electromagnetic fields, at the interfaces between the different domains.

\subsection{Harmonic Maxwell equations}

Maxwell equations in the harmonic regime read

$$
\left\{\begin{array}{l}
\nabla \times \hat{E}-i \omega \mu \hat{H}=0 \\
\nabla \times \hat{H}+i \omega \epsilon \hat{E}=0
\end{array}\right.
$$

where $\omega$ is the frequency, $\mu$ and $\epsilon$ are respectively the magnetic permeability and the electric permittivity and $\hat{E}$ and $\hat{H}$ stand for the (time Fourier transform of the) electric and magnetic fields. Notice that we use the time convention where the electric and magnetic fields are sought as $\exp (-i \omega t) \hat{E}$ and $\exp (-i \omega t) \hat{H}$ respectively. Introducing the wavenumber $k=\frac{\omega}{c}$ and wave speed $c=\frac{1}{\sqrt{\epsilon \mu}}$ together with the dimensionless quantities

$$
E=\sqrt{\epsilon} \hat{E}, H=\sqrt{\mu} \hat{H}
$$

we may rewrite Maxwell equations as

$$
\left\{\begin{array}{l}
\nabla \times E-i k H=0 \\
\nabla \times H+i k E=0
\end{array}\right.
$$

We assume that the three electromagnetic fields $\left(E_{\mathrm{inc}}, H_{\mathrm{inc}}\right),\left(E_{\mathrm{sc}}, H_{\mathrm{sc}}\right)$ and $\left(E_{\mathrm{tr}}, H_{\mathrm{tr}}\right)$ satisfy those equations, while the physicality of electromagnetic scattered fields in the unbounded domain is enforced through the SilverMuller radiation condition

$$
\left|E_{\mathrm{sc}}-H_{\mathrm{sc}} \times \frac{\vec{r}}{r}\right| \leq \frac{c}{r^{2}} \text { for } r \rightarrow+\infty
$$

The equations for each of the electric and magnetic fields are obtained by taking the curl of both equations in (3) and substitute back with the other one. One obtains

$$
\left\{\begin{aligned}
\nabla \times \nabla \times E-k^{2} E & =0 \\
\nabla \times \nabla \times H-k^{2} H & =0
\end{aligned}\right.
$$

Eventually, we define the (dimensionless) electric and magnetic currents on the surface of the scattering object by

$n$ being the outer normal to the surface.

$$
\left\{\begin{aligned}
J & =H_{\text {tot }} \times n \\
M & =E_{\text {tot }} \times n
\end{aligned}\right.
$$

\subsection{Integral Representation Theorem and Integral Equations}

The heart of the BEM lies in the integral representation of an electromagnetic field $(E, H)$ that solves $(3)$ in $\Omega^{+}$and inside $\Omega^{-}=\mathbb{R}^{3} \backslash \overline{\Omega^{+}}$, and which satisfies furthermore the Silver-Muller condition (4). One has, calling $\Gamma=\partial \Omega^{+}$the surface of the object (see e.g. $[5,6,15,22]$ )

$$
\left(\begin{array}{c}
E \\
H
\end{array}\right)=\left(\begin{array}{cc}
\mathcal{K}_{k} & \mathcal{T}_{k} \\
-\mathcal{T}_{k} & \mathcal{K}_{k}
\end{array}\right)\left(\begin{array}{c}
{[E] \times n} \\
{[H] \times n}
\end{array}\right)
$$


where the boundary operators $\mathcal{T}_{k}$ and $\mathcal{K}_{k}$, are given by

$$
\begin{aligned}
\mathcal{T}_{k} u(y) & :=i k \int_{\Gamma} G_{k}(x-y) u(x) \mathrm{d} \gamma(x)+\frac{i}{k} \nabla \int_{\Gamma} G_{k}(x-y) \nabla_{\Gamma} \cdot u(x) \mathrm{d} \gamma(x), \\
\mathcal{K}_{k} u(y) & :=\nabla \times \int_{\Gamma} G_{k}(x-y) u(x) \mathrm{d} \gamma(x)
\end{aligned}
$$

and the relation (7) only holds for any $y \notin \Gamma$.

In (8) and (9), we have also denoted by

$$
G_{k}(r)=\frac{1}{4 \pi r} e^{i k r}
$$

the free space Helmholtz Green kernel in $\mathbb{R}^{3}$, and by [.] the jump

$$
[u]:=\gamma^{-} u-\gamma^{+} u
$$

where the trace operators $\gamma^{ \pm}$are given by

$$
\gamma^{ \pm} u(y):=\lim _{x \rightarrow y, x \in \Omega^{ \pm}} u(x), \quad y \in \Gamma
$$

and $n$ is the unit outer normal to $\Gamma$.

Defining eventually for $y \in \Gamma$ the boundary operators

$$
\begin{aligned}
T_{k} u(y) & :=i k \int_{\Gamma} G_{k}(x-y) u(x) \mathrm{d} \gamma(x)+\frac{i}{k} \nabla \int_{\Gamma} G_{k}(x-y) \nabla_{\Gamma} \cdot u(x) \mathrm{d} \gamma(x), \\
K_{k} u(y) & :=\nabla \times \int_{\Gamma} G_{k}(x-y) u(x) \mathrm{d} \gamma(x),
\end{aligned}
$$

we also have that those operators satisfy (if the surface $\Gamma$ is smooth enough)

$$
\begin{aligned}
\gamma^{ \pm}\left(\mathcal{T}_{k} u\right) & =T_{k} u, \\
\gamma^{ \pm}\left(\mathcal{K}_{k} u\right) & =K_{k} u \mp \frac{1}{2} n \times u
\end{aligned}
$$

while the traces of the electromagnetic field obey

$$
\gamma^{ \pm}\left(\begin{array}{l}
E \\
H
\end{array}\right)=\left(\begin{array}{cc}
K_{k} \mp \frac{1}{2} n \times & T_{k} \\
-T_{k} & K_{k} \mp \frac{1}{2} n \times
\end{array}\right)\left(\begin{array}{c}
{[E] \times n} \\
{[H] \times n}
\end{array}\right) .
$$

A priori, the previous formula only applies when the wavenumber $k$ (which depends on the permittivity $\epsilon$ and the permeability $\mu$ ) is identical in $\Omega^{+}$and $\Omega^{-}$. This is not the case in our problem for which there exists three domains $\left(\Omega^{+}, \Omega_{\mathrm{PEC}}^{-}, \Omega_{\mathrm{d}}^{-}\right)$with, for each, different parameters. The application of the preceding formalism to our context is precisely the subject of the following sub-section.

\subsection{Boundary formulation at the surface of the object}

In order to be able to use the previous results, we choose to prolong the exterior scattered field $\left(E_{\mathrm{sc}}, H_{\mathrm{sc}}\right)$ by the opposite of the incident field $\left(-E_{\text {inc }},-H_{\text {inc }}\right)$ which is a particular solution of Maxwell's equations. Introducing the electric and magnetic currents on $\Gamma$

$$
J=H_{\mathrm{tot}} \times n=\left(H_{\mathrm{sc}}+H_{\mathrm{inc}}\right) \times n, \quad M=E_{\mathrm{tot}} \times n=\left(E_{\mathrm{sc}}+E_{\mathrm{inc}}\right) \times n,
$$


and taking the interior trace in the integral equations (17) we readily obtain that $(M, J)$ satisfies

$$
\left(\begin{array}{cc}
K_{k}+\frac{1}{2} n \times & T_{k} \\
-T_{k} & K_{k}+\frac{1}{2} n \times
\end{array}\right)\left(\begin{array}{c}
-M \\
-J
\end{array}\right)=\left(\begin{array}{c}
-E_{\text {inc }} \\
-H_{\text {inc }}
\end{array}\right) .
$$

Notice that on the interface $\Gamma_{\mathrm{PEC}}$ between the vacuum $\Omega^{+}$and the PEC domain $\Omega_{\mathrm{PEC}}^{-}$the equations can be simplified by using the characteristics of the PEC material. Indeed, there is no electric field $E_{\text {tot }}=0$ inside $\Omega_{\mathrm{PEC}}^{-}$. The continuity of the tangential component of the electric field at the interface $\Gamma_{\mathrm{PEC}}$ then yields $M=0$ on this interface.

Anticipating on the following, and for purposes of conditioning in the system to solve, we combine both equations in (19) and consider only $J$ as the unknown. We obtain

$$
\left(\alpha T_{k}+\beta\left(n \times K_{k}-\frac{1}{2} \mathrm{Id}\right)\right) J=F,
$$

for two real $\alpha, \beta \in \mathbb{R}$ and where we introduced

$$
F=\alpha E_{\mathrm{inc}}+\beta n \times H_{\mathrm{inc}}-\left(\alpha\left(K_{k}+\frac{1}{2} n \times\right)-\beta n \times T_{k}\right) M .
$$

The quantity $M$ (which is only non-zero on $\Gamma_{\mathrm{d}}$ ) will be later determined from the coupling with the dielectric interior domain.

Multiplying (20) by a test function $J^{\prime}$ to reformulate this equation in a variational form leads, after integration on $\Gamma$, to

$$
\int_{\Gamma}\left(\alpha T_{k}+\beta\left(n \times K_{k}-\frac{1}{2} \mathrm{Id}\right)\right) J \cdot J^{\prime} \mathrm{d} \gamma=\int_{\Gamma} F \cdot J^{\prime} \mathrm{d} \gamma
$$

This is the equation, written only in the unknown $J$, that we will consider at the interface $\Gamma=\Gamma_{\mathrm{d}} \cup \Gamma_{\mathrm{PEC}}$. The evaluation of the right-hand-side, and its dependence with $M$ will be made clear later.

\subsection{Volumic formulation in the dielectric domain}

We now obtain a variational formulation for the interior dielectric domain. Using Maxwell's equation for the electric field $E_{\mathrm{tr}}$ in (5), multiplying by a test function $E^{\prime}$ and integrating by parts over $\Omega_{\mathrm{d}}^{-}$gives

$$
\begin{aligned}
\int_{\Omega_{\mathrm{d}}^{-}}\left(\nabla \times E_{\mathrm{tr}}\right) \cdot\left(\nabla \times E^{\prime}\right)-k_{d}^{2} E_{\mathrm{tr}} \cdot E^{\prime} \mathrm{d} \Omega & =-\int_{\Gamma_{\mathrm{d}}}\left[n \times\left(\nabla \times E_{\mathrm{tr}}\right)\right] \cdot E^{\prime} \mathrm{d} \gamma \\
& =i k_{d} \int_{\Gamma_{\mathrm{d}}} J_{\mathrm{tr}} \cdot E^{\prime} \mathrm{d} \gamma
\end{aligned}
$$

using the first equation in (3), and where we have denoted by $J_{\mathrm{tr}}=H_{\mathrm{tr}} \times n$ the electric interior current.

Note that the boundary integral on the right-hand side of the equation is taken only on $\Gamma_{\mathrm{d}}$. This is justified by the continuity of the tangential component of $E_{\text {tr }}$ on the interface $\Sigma$ which imposes $E_{\operatorname{tr}} \times n=0$ on $\Sigma$ and therefore does not require testing.

Equation (23) is solved in the interior of the dielectric domain to compute the unknown $E_{\text {tr }}$ knowing the boundary current $J_{\mathrm{tr}}$.

\subsection{Coupling}

The final coupling between the boundary and volumic formulations is obtained by considering the transmission conditions at the interface between the different domains. The continuity of the tangential components of the electric and magnetic fields on the surface $\Gamma_{\mathrm{d}}$ can be written as 


$$
\begin{aligned}
& \gamma^{-}\left(E_{\mathrm{tr}} \times n\right)=\sqrt{\epsilon_{r}} \gamma^{+}\left(\left(E_{\mathrm{sc}}+E_{\mathrm{inc}}\right) \times n\right), \quad \text { on } \Gamma_{\mathrm{d}} \text {, } \\
& \gamma^{-}\left(H_{\mathrm{tr}} \times n\right)=\sqrt{\mu_{r}} \gamma^{+}\left(\left(H_{\mathrm{sc}}+H_{\mathrm{inc}}\right) \times n\right), \quad \text { on } \Gamma_{\mathrm{d}} \text {. }
\end{aligned}
$$

Recall that we have chosen to prolong the exterior field $\left(E_{\mathrm{sc}}, H_{\mathrm{sc}}\right)$ by the opposite of incident field $\left(-E_{\mathrm{inc}},-H_{\mathrm{inc}}\right)$ this can be rewritten readily as

$$
E_{\mathrm{tr}} \times n=\sqrt{\epsilon_{r}} M, \quad \text { and } \quad J_{\mathrm{tr}}=\sqrt{\mu_{r}} J
$$

Equations (22) and (23), with the two previous coupling relations, form a complete system with the two unknowns $J$ and $E_{\mathrm{tr}}$. The right-hand side of equation (23) can be rewritten to obtain the final interior equation as

$$
\int_{\Omega_{\mathrm{d}}^{-}}\left(\nabla \times E_{\mathrm{tr}}\right) \cdot\left(\nabla \times E^{t}\right)-k_{d}^{2} E_{\mathrm{tr}} \cdot E^{\prime} \mathrm{d} \Omega=\sqrt{\mu_{r}} i k_{d} \int_{\Gamma_{\mathrm{d}}} J \cdot E^{\prime} \mathrm{d} \gamma
$$

The right-hand-side of equation (22) can be rewritten to obtain the final boundary equation as

$$
\begin{aligned}
\int_{\Gamma_{\mathrm{d}} \cup \Gamma_{\mathrm{PEC}}} & \left(\alpha T_{k}+\beta\left(n \times K_{k}-\frac{1}{2} \mathrm{Id}\right)\right) J \cdot J^{\prime} \mathrm{d} \gamma \\
= & \int_{\Gamma_{\mathrm{d}} \cup \Gamma_{\mathrm{PEC}}}\left(\alpha E_{\mathrm{inc}}+\beta n \times H_{\mathrm{inc}}\right) \cdot J^{\prime} \mathrm{d} \gamma \\
& -\frac{1}{\sqrt{\epsilon_{r}}} \int_{\Gamma_{\mathrm{d}} \cup \Gamma_{\mathrm{PEC}}}\left(\left(\alpha\left(K_{k}+\frac{1}{2} n \times\right)-\beta n \times T_{k}\right)\left(E_{\mathrm{tr}} \times n\right)\right) \cdot J^{\prime} \mathrm{d} \gamma
\end{aligned}
$$

\subsection{Discretization and solving strategy}

The solving strategy involves formally assembling two systems:

- The first system arises from the volumic FEM discretization of Maxwell equations for the electric field inside the dielectric region as given by equation (27). This is a sparse system.

- The second system and arises from the BEM discretization of the scattering problem on $\Gamma$ as given by equation (28). This is a dense system. However, instead of assembling the dense matrix, the fast SCSD [1] method is used to speed-up the computations.

To properly discretize the equations, the electric current $J$ is represented using (div-conforming) Rao-WiltonGlisson finite elements while the electric field $E_{\mathrm{tr}}$ is represented by using (rot-conforming) Nédélec edge finite elements on tetrahedra.

The coupled system $(27,28)$ in the two unknowns $J$ and $E_{\operatorname{tr}}$ is solved iteratively using a Schur complement approach. The main unknown $J$ is used as the iterate and the field $E_{\mathrm{tr}}$ is computed exactly at each iteration using MATLAB's mldivide function (backslash), which amounts to solve the underlying system by a LU factorization. The iterative solver is a Multi Generalized Conjugate Residual (MGCR) algorithm [17,18], allowing for simultaneous resolution of all incidences and polarisations for a given frequency. The algorithm is not restarted during convergence. We moreover use as preconditioner of the system the sparse local correction matrix arising in the SCSD method, after performing an incomplete LU decomposition. Eventually, in our numerical computations we take $\alpha=-0.5$ and $\beta=1$ in (28). 


\section{Sparse Cardinal Sine Decomposition}

As explained in $[1,2,4]$ the SCSD is a method that is suitable for compressing matrices coming from the discretization of convolution kernels like the ones coming from the discretization of $T_{k}$ and $K_{k}$. For the computation presented before, we need to use the Helmholtz kernel

$$
G(r)=\frac{e^{i k r}}{4 \pi r}
$$

Following the strategy developed in [1], we therefore expand the kernel as a series of cardinal sines

$$
\frac{e^{i k r}}{4 \pi r} \sim \sum_{p} \alpha_{p} \frac{\sin \left(\lambda_{p} r\right)}{r}
$$

which is accurate to within a given precision, with $r$ in a given interval $\left[r_{\min }, r_{\max }\right]$, and where the weights $\left(\alpha_{p}\right)_{p}$ and the frequencies $\left(\lambda_{p}\right)_{p}$ need to be chosen. Let us notice that the choice of $r_{\max }$ comes from the maximal interaction distance between two points in the mesh, or in other words, an upper bound of the diameter of the object. The parameter $r_{\min }$, instead, is tuned in order to optimize local versus global computations. Indeed, the formula (29) being inaccurate for $r<r_{\min }$, one needs to compute a (sparse) correction matrix that handles the interactions between points that are at a distance smaller to $r_{\min }$. Depending on the choice of $r_{\min }$, the local matrix will be bigger (for larger $r_{\text {min }}$ ) while the expansion (29) possesses less terms. On the other hand, for smaller $r_{\min }$ the series (29) contains more terms leading to an increasing cost of the evaluation of large interactions, while the local corrections are reduced. The fine tuning of $r_{\text {min }}$ that leads to a numerical cost for computing local corrections that is comparable to the one for computing interactions at large distances, is explained in $[1,2,4]$.

For Helmholtz kernel, it turns out that a good strategy consists in considering the real part and the imaginary part separately and writing

$$
\cos (k r) \sim \sum_{p=0}^{P-1} \alpha_{p}(\sin ((k+(2 p+1) \delta) r)-\sin ((k-(2 p+1) \delta) r))
$$

where $\delta=\frac{\pi}{r_{\min }+r_{\max }}$. It can be shown (see [1]) that the above formula is valid to within a numerical precision $\varepsilon$ if $P=-\frac{\log (\varepsilon) r_{\max }}{2 r_{\min }}$, and the coefficients $\left(\alpha_{p}\right)_{0 \leq p<P}$ are computed in a least square sense, that is to say, by minimizing

$$
\int_{r_{\min }}^{r_{\max }}\left(\cos (k r)-\sum_{p=0}^{P-1} \alpha_{p}(\sin ((k+(2 p+1) \delta) r)-\sin ((k-(2 p+1) \delta) r))\right)^{2} d r
$$

Notice that this amounts to solve a linear system.

For $P$ small enough, the above procedure gives a family of frequencies $\left(\lambda_{p}\right)$ symmetrically distributed around $k$. If $P$ is too big (namely when one desires a very high precision or the quotient $r_{\max } / r_{\min }$ is very large), the frequencies that appear in formula (30) are likely to become negative. To simplify the formula, we thus restrict $\delta$ to be of the form $\delta=\frac{k}{2 N}$ for an integer $N<P$, and make use of the fact that the sine is an odd function. This allows us to rewrite equivalently (30) as

$$
\cos (k r) \sim \sum_{p=0}^{N+P-1} \alpha_{p}^{\prime} \sin \left(\frac{2 p+1}{2 N} k r\right)
$$


in which only $N+P$ coefficients $\left(\alpha_{p}^{\prime}\right)_{0 \leq p \leq N+P-1}$ appear (instead of $2 P$ ). These coefficients can still be computed using the least square procedure explained above.

Once (30) is obtained, we have a decomposition of the kernel as

$$
\frac{e^{i k r}}{r} \sim \sum_{p=0}^{N+P-1} \alpha_{p}^{\prime}\left(\frac{\sin \left(\frac{2 p+1}{2 N} k r\right)}{r}\right)+i \frac{\sin k r}{r}
$$

which is nothing than a decomposition in cardinal sines. This enables, following the strategy of $[1,2,4]$ to write the discrete convolution kernel using the Type 3 Non Uniform FFT $[10,12,19]$, at a computational cost proportional to $N \log (N)$ where $N$ is the number of unknowns.

\section{Numerical RESUlts}

In this section, a number of numerical examples are presented in order to validate and demonstrate the performance of the proposed method for solving electromagnetic scattering problems for obstacles composed both of PEC and dielectric materials. The software used for the numerical computations is a MATLAB code referred to as MyBEM. It includes a full BEM and FEM code with suitable finite elements for electromagnetic scattering (Raviart-Thomas and Nédélec finite elements) and an implementation of the SCSD method.

\subsection{Validation}

The first example shown here serves as validation and consists in the scattering of a $300-\mathrm{MHz}$ plane wave by a sphere of radius $R=0.8 \mathrm{~m}$. Two subcases are considered: the PEC case and the dielectric-layer case. For the latter, there is an absorbing dielectric material with relative permittivity $\epsilon_{r}=1.5+0.1 i$ and relative permeability $\mu_{r}=2.5+1.8 i$ between the radius $R=0.8 \mathrm{~m}$ and the radius $R=1 \mathrm{~m}$. The two configurations do not correspond exactly to the equations we have written previously, but are special cases. Our interest here is to only validate the software by comparing to analytical solutions.

The validation consists in evaluating the bistatic radar cross section (RCS) of these two objects. Given a viewing direction $r$, the RCS in square meters is defined as

$$
\operatorname{RCS}:=\lim _{|r| \rightarrow \infty} 4 \pi r^{2} \frac{\left|E_{\mathrm{sc}}(r)\right|^{2}}{\left|E_{\mathrm{inc}}(r)\right|^{2}} .
$$

The bistatic RCS is computed from this definition by keeping the incident direction fixed and varying the viewing direction $r$. To be able to compute this quantity from the numerical solutions one need to evaluate the scattered electric field $E_{\mathrm{sc}}(r)$ for large values of $|r|$. In the far field, one can obtain an asymptotic expansion of the integral representation (19). The electric field $E_{\mathrm{sc}}$ reads, for large arguments $|r|$,

$$
\begin{aligned}
E_{\mathrm{sc}}(r) & =\frac{e^{i k|r|}}{|r|} E_{\mathrm{sc}}^{\infty}\left(\frac{r}{|r|}\right)+\mathcal{O}\left(\frac{1}{|r|^{2}}\right) \\
E_{\mathrm{sc}}^{\infty} & =\mathcal{K}_{k}^{\infty} M+\mathcal{T}_{k}^{\infty} J
\end{aligned}
$$

with

$$
\begin{aligned}
\mathcal{T}_{k}^{\infty} u(r) & :=-\frac{i k}{4 \pi} r \times \int_{\Gamma} e^{-i k r \cdot x} u(x) \times r \mathrm{~d} \gamma(x), \\
\mathcal{K}_{k}^{\infty} u(r) & :=-\frac{i k}{4 \pi} r \times \int_{\Gamma} e^{-i k r \cdot x} u(x) \mathrm{d} \gamma(x),
\end{aligned}
$$

and $M$ and $J$ are solutions of the integral equation considered. 
The bistatic RCS as a function of the angle $\alpha$ between the incident and viewing directions is reported in Figures 2 and 3 for the two subcases and two polarisations (vertical VV and horizontal HH). We note the perfect agreement between the bistatic RCS computed with the numerical solutions and the analytic solutions.

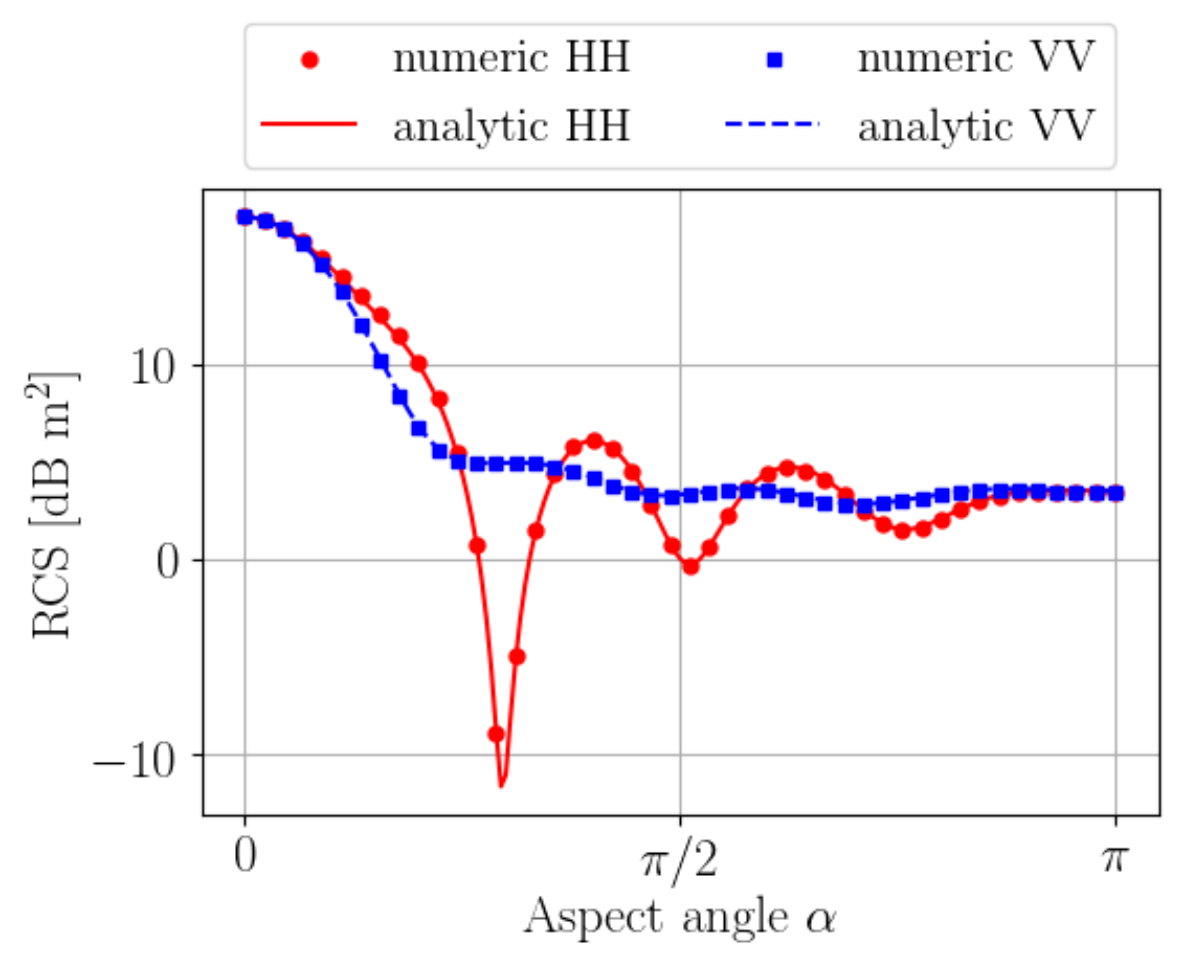

FiguRE 2. Bistatic RCS of the scattering of a $300-\mathrm{MHz}$ plane wave by a PEC sphere of radius $R=0.8 m$ for $\mathrm{VV}$ and $\mathrm{HH}$ polarisations.

We now turn to another validation case that falls exactly in the category of problems we previously described. The geometry used is a cylinder aligned with the $x$-axis, with radius $R=0.0381 \mathrm{~m}$ and total length $L=0.1524$ $m$. The portion of the cylinder with positive $x$-coordinates is a PEC of length $a=0.1016 \mathrm{~m}$, while the other portion is a dielectric material of length $b=0.0508 \mathrm{~m}$. The dielectric material has a relative permittivity $\epsilon_{r}=2.6$ and relative permeability $\mu_{r}=1$. The obstacle is lit by a 3 -Ghz plane wave and we compute the monostatic RCS for two polarisations.

The monostatic RCS of the electromagnetic scattering by this obstacle is computed from the definition (34) of the RCS with incident direction and viewing direction $r$ opposite to each other (transmitter and receiver co-located). The monostatic RCS as a function of the angle between the $x$-axis and the viewing direction for the two polarisations is reported in Figures 4 and 5. The computed RCS are again in very good agreement with the results given in the literature [14].

\subsection{ISAE workshop test case}

We now give a description of the results obtained on the test case number 3 of the workshop ISAE 2016, entitled RCS and radar imaging for an UAV-like with two RAM regions. The test case consists in computing the electromagnetic scattering of an UAV-like object. The UAV is assumed to be a perfect electric conductor 


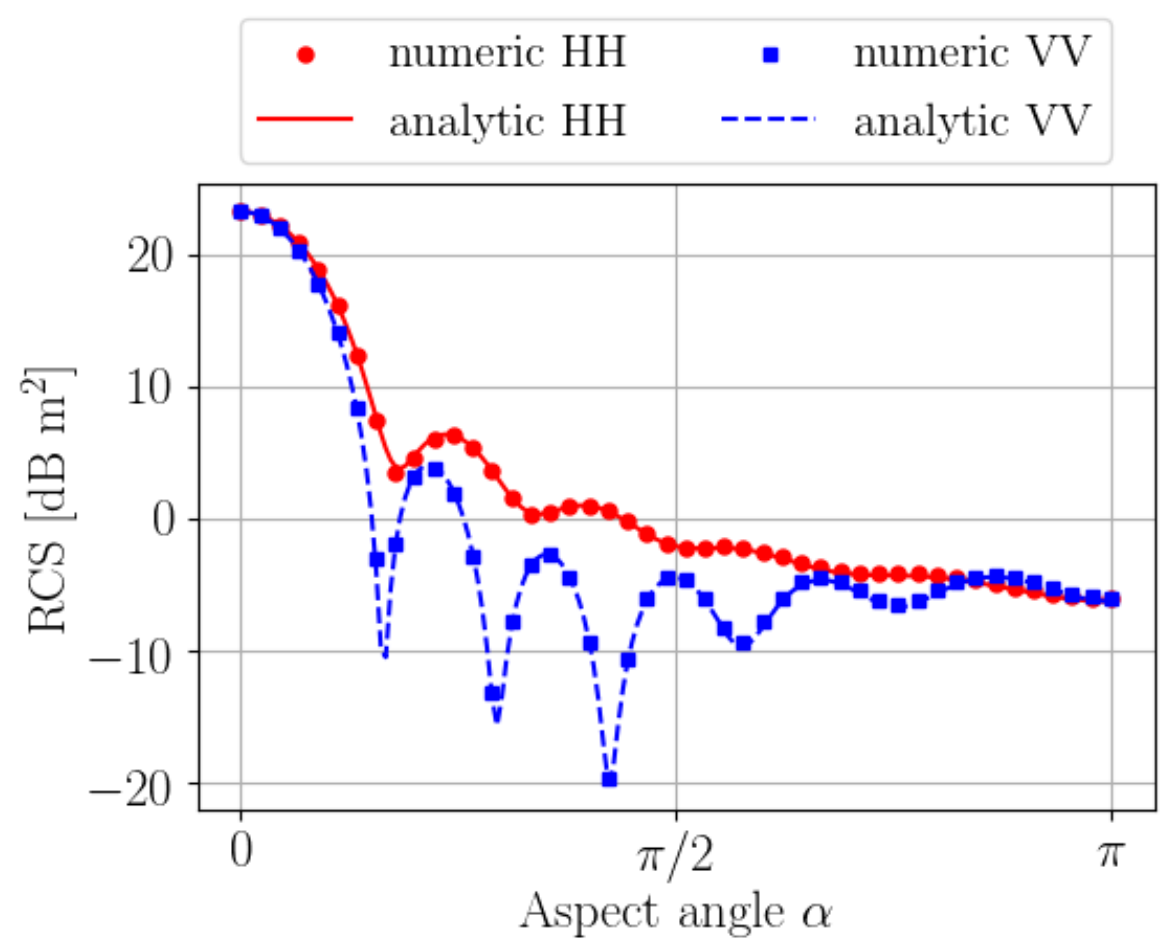

FiguRE 3. Bistatic RCS of the scattering of a $300-\mathrm{MHz}$ plane wave by a PEC sphere with an absorbing dielectric layer between the radius $R=0.8 m$ and the radius $R=1 m$ for $\mathrm{VV}$ and HH polarisations.

(PEC) except in two regions, the nose and a slot, which are filled with an absorbing material with relative permittivity $\epsilon_{r}=1.5+0.1 i$ and relative permeability $\mu_{r}=2.5+1.8 i$. The geometry of the object is rather complicated and contains many small details, making the test case both interesting and challenging.

The surface mesh was provided by J. Simon from Onera. The two volume regions were constructed from this surface mesh, using the software GMSH [13]. In our simulations, the mesh of the UAV is composed of:

- Two volume meshes discretizing the two dielectric regions, containing 80135 tetrahedra in total;

- One surface mesh for the exterior interface $\Gamma_{\mathrm{d}} \cup \Gamma_{\text {PEC }}$ between the vacuum and the obstacle, and containing 192604 triangles in total;

- Two surface meshes for the interfaces $\Sigma$ between the two dielectric regions and the PEC region, and containing 3052 triangles in total.

The mesh is represented in Figure 6, using GMSH. A close-up view of the nose of the aircraft is given in Figure 7.

There are 288906 degrees of freedom in total for the unknown $J, 101178$ degrees of freedom in total for the unknown $E_{\mathrm{tr}}, 16578$ of which are on the exterior surface $\Gamma_{\mathrm{d}}$.

For the target described above, the monostatic RCS is simulated:

- for the frequency range $f=2.5$ to $4.0 \mathrm{GHz}$, with $\delta f=30 \mathrm{MHz}$ (i.e. 51 frequencies),

- for the azimuth angle $\Phi=-15$ to $15 \mathrm{deg}$, with $\delta \Phi=0.5 \mathrm{deg}$ (i.e. 61 angles),

- for $\theta=100 \mathrm{deg}$,

- for both polarizations (VV-polarization and HH-polarization). 


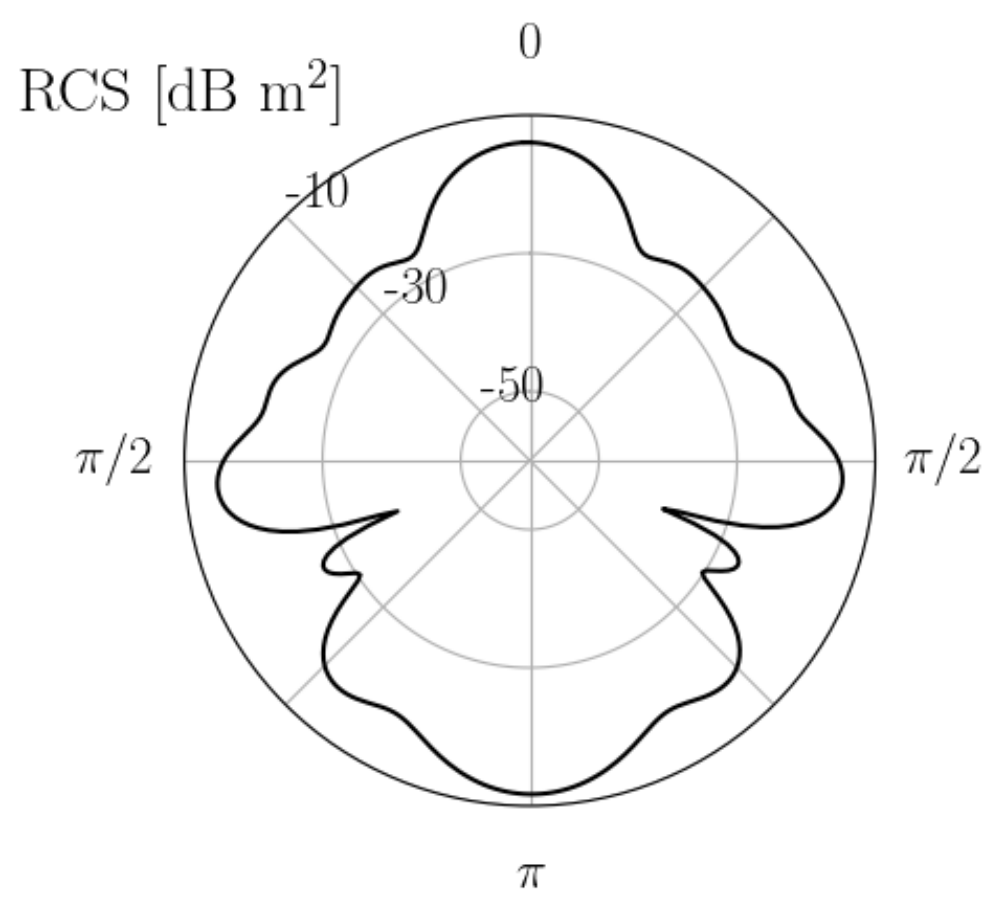

FiguRE 4. Monostatic RCS of the scattering of a $3-\mathrm{GHz}$ plane wave by a cylinder partially composed of a dielectric material. VV polarisation.

The frequency-mean monostatic RCS is given in Figure 8 for the two polarisations. The Inverse Synthetic Aperture Radar (ISAR) image is also computed from the monostatic data. We define it here for $-2 \leq x \leq 2.5$ and $-2 \leq y \leq 2$ as

$$
\operatorname{ISAR}(x, y)=\sum_{\Phi} \sum_{f} E_{\mathrm{sc}}(\Phi, f) e^{i \frac{2 \pi f}{c} 2 r(x, y)}
$$

with

$$
r(x, y)=\sqrt{(x-R \cos \Phi)^{2}+(y+R \sin \Phi)^{2}},
$$

where $R$ is here set arbitrary to 100. The ISAR images for both polarisations are provided in Figures 9 and 10 . The results presented are in good agreement with those obtained by the other participants of the workshop.

The results given are obtained on two different machines which characteristics are given in Table 1 . The use of the machine in the second column in Table 1 was required to be able to address the highest frequencies (from $3.58 \mathrm{GHz}$ up to $4 \mathrm{GHz}$ ) which are more memory consuming.

Some performance information on the resolution of the electromagnetic scattering by the UAV are given in Table 2. The performance data depends on the frequency considered and the machine used, hence justifying the fact that we only provide ranges. The runs on the first machine are slightly faster, albeit the slower clock rate, than the runs on the second one. This is likely explained by the different MATLAB version. We finally note that if these computations had been done sequentially on these two machines, we arrive at a total computing time of around 24 days for the whole test case. 


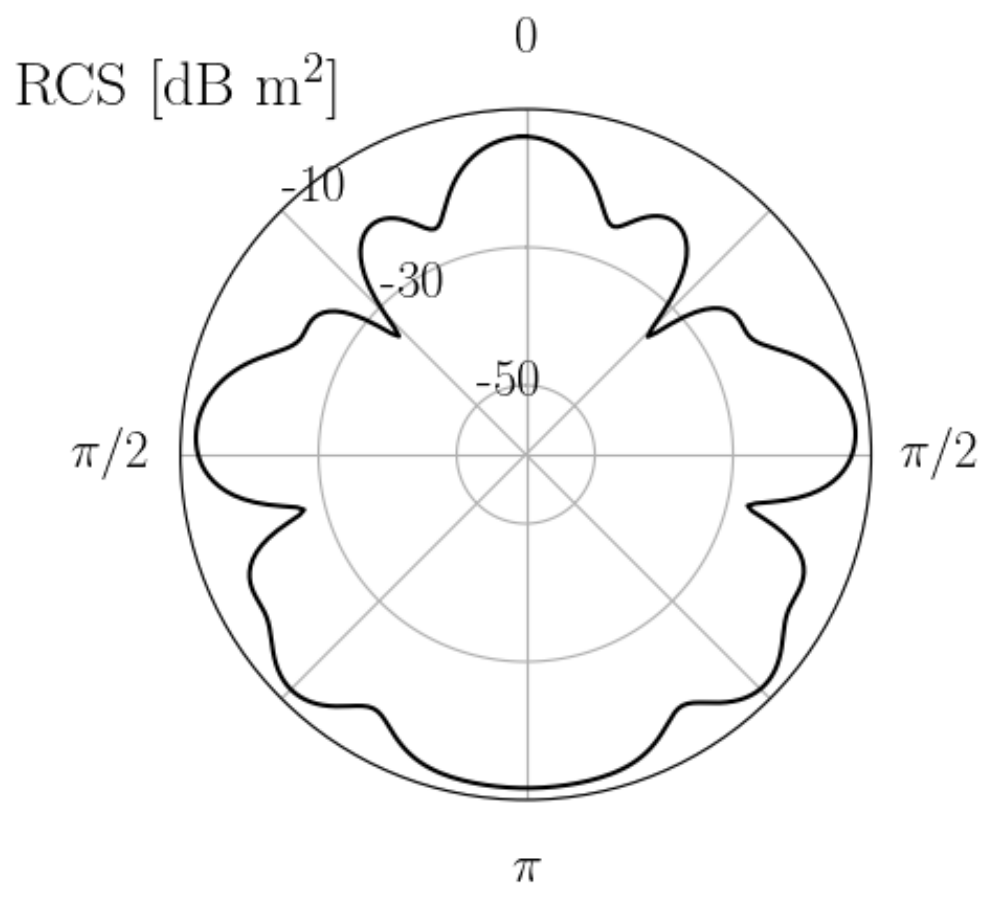

FiguRE 5. Monostatic RCS of the scattering of a $3-\mathrm{GHz}$ plane wave by a cylinder partially composed of a dielectric material. HH polarisation.

\begin{tabular}{c|c|c} 
& Machine 1 & Machine 2 \\
\hline Processor & Intel Xeon E5-2650 v2 & Intel Xeon E5-2667 v3 \\
CPUs Max/Used & $16 / 12$ & $16 / 12$ \\
Cache & $20 \mathrm{M}$ & $20 \mathrm{M}$ \\
Frequency & $2.6 \mathrm{GHz}$ & $3.2 \mathrm{GHz}$ \\
Memory & $64 \mathrm{Go}$ & $128 \mathrm{Go}$ \\
MATLAB version & R2015b & R2013a
\end{tabular}

TABLE 1. Machine and software characteristics.

\begin{tabular}{c|c|c} 
& Machine 1 & Machine 2 \\
\hline Frequency range & $2.5-3.55 \mathrm{GHz}$ & $3.58-4 \mathrm{GHz}$ \\
Assembling time & $20-40 \mathrm{~min}$ & $55-80 \mathrm{~min}$ \\
Iteration count & $524-741$ & $717-813$ \\
Time per iteration & $\sim 50 \mathrm{~s}$ & $\sim 60 \mathrm{~s}$ \\
Total & $8-11 \mathrm{~h}$ & $13.5-15.5 \mathrm{~h}$
\end{tabular}

TABLE 2. Iteration information and timings per resolution/frequency (122 RHS).

\section{Conclusion}

We presented in this paper an approach to solve FEM-BEM coupling problems for electromagnetism scattering by obstacles composed of different materials: a perfect electric conductor and a dielectric. We reformulated 


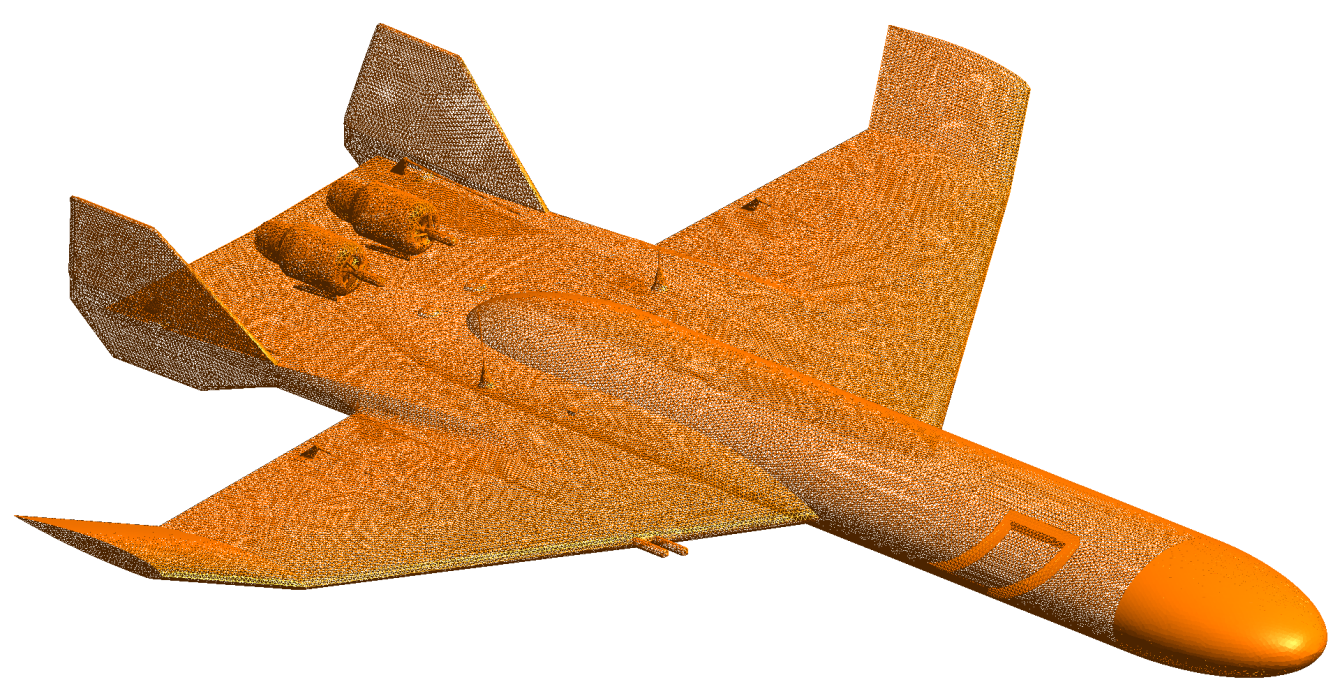

Figure 6. The mesh of the UAV that was used, with the two volumic regions.

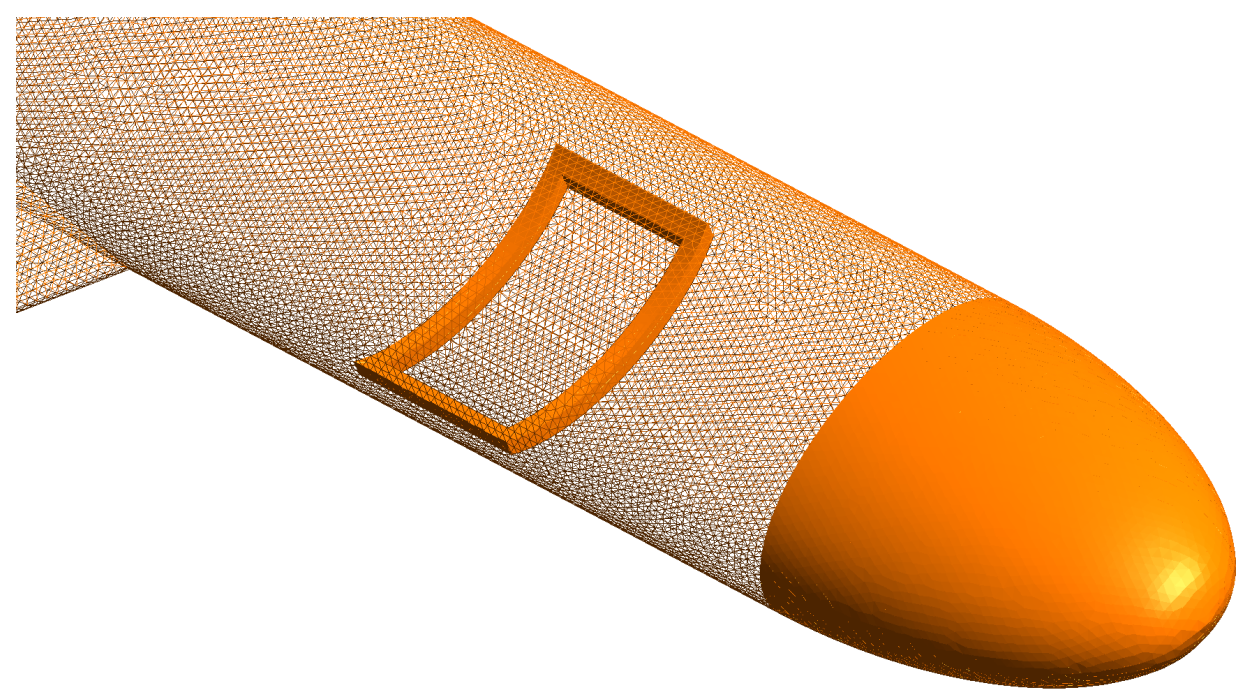

Figure 7. The mesh of the UAV. Close-up view on the nose

the problem with two unknowns, the electric current on the surface of the scatterer and the electric field inside the dielectric material, coupled in two equations discretised respectively via the boundary element method and the volumic finite element method. The BEM part is efficiently computed by using the novel Sparse Cardinal Sine Decomposition method. A test case of industrial interest has been successfully solved using the proposed approach which has been implemented in a MATLAB code. 


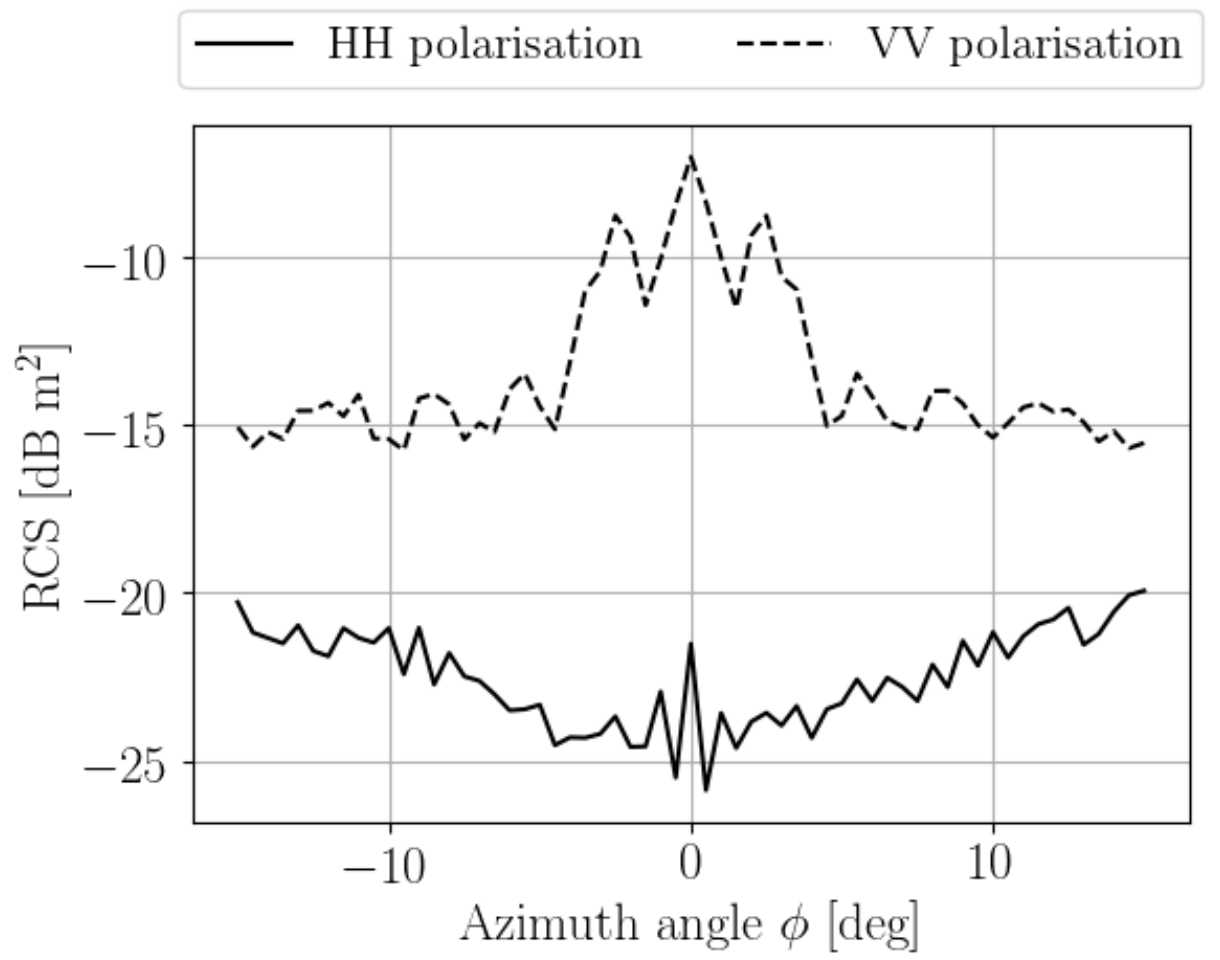

FIGURE 8. Mean monostatic RCS of the UAV-like object.

\section{REFERENCES}

[1] F. Alouges and M. Aussal, The Sparse Cardinal Sine Decomposition and its application for fast numerical convolution, Numerical Algorithms, 1-22, 2014.

[2] F. Alouges And M. Aussal, The Sparse Cardinal Sine Decomposition applied to three-dimensional acoustic scattering, In preparation.

[3] F. Alouges, M. Aussal, A. Lefebvre-Lepot, F. Pigeonneau and A. Sellier, The Sparse Cardinal Sine Decomposition applied to Stokes integral equations, Proceedings of the International Conference on Multiphase Flow, ICMF 2016, Firenze, Italy, May 22 - 27, 2016.

[4] M. Aussal, Méthodes numériques pour la spatialisation sonore, de la simulation à la synthèse binaurale, $\mathrm{PhD}$ thesis, Ecole polytechnique, 2014.

[5] A. Bendali, Equations intgrales en lectromagntisme, available at https://www.math.univtoulouse.fr $\sim^{\sim}$ abendali/polyelec1314.pdf .

[6] D. Colton and R. KRess, Integral Equation Methods in Scattering Theory. John Wiley \& Sons (1983).

[7] A. Dutt And V. Rokhlin, Fast Fourier transforms for nonequispaced data, SIAM Journal of Scientific Computing, $14,1993$.

[8] L. Greengard and V. Rokhlin, A fast algorithm for particle simulations, Journal of Computational Physics, 73(2):325-348, 1987.

[9] L. Greengard, The rapid evaluation of potential fields in particle systems, MIT press, 1988.

[10] L. Greengard and J. Y. Lee, Accelerating the non-uniform Fast Fourier Transform, SIAM review, 46(3):443-455, 2004.

[11] W. Hackbusch, A sparse matrix arithmetic based on $\mathcal{H}$-matrices. Part I: introduction to $\mathcal{H}$-matrices, Computing, 62(2):89$108,1999$.

[12] J. Y. LeE And L. Greengard, The type 3 non-uniform FFT and its applications, Journal of Computational Physics, 206:1 (2005), 1-7.

[13] C. Geuzaine and J.-F. Remacle, Gmsh: a three-dimensional finite element mesh generator with built-in pre- and postprocessing facilities, International Journal for Numerical Methods in Engineering 79(11), (2009), 1309-1331. 


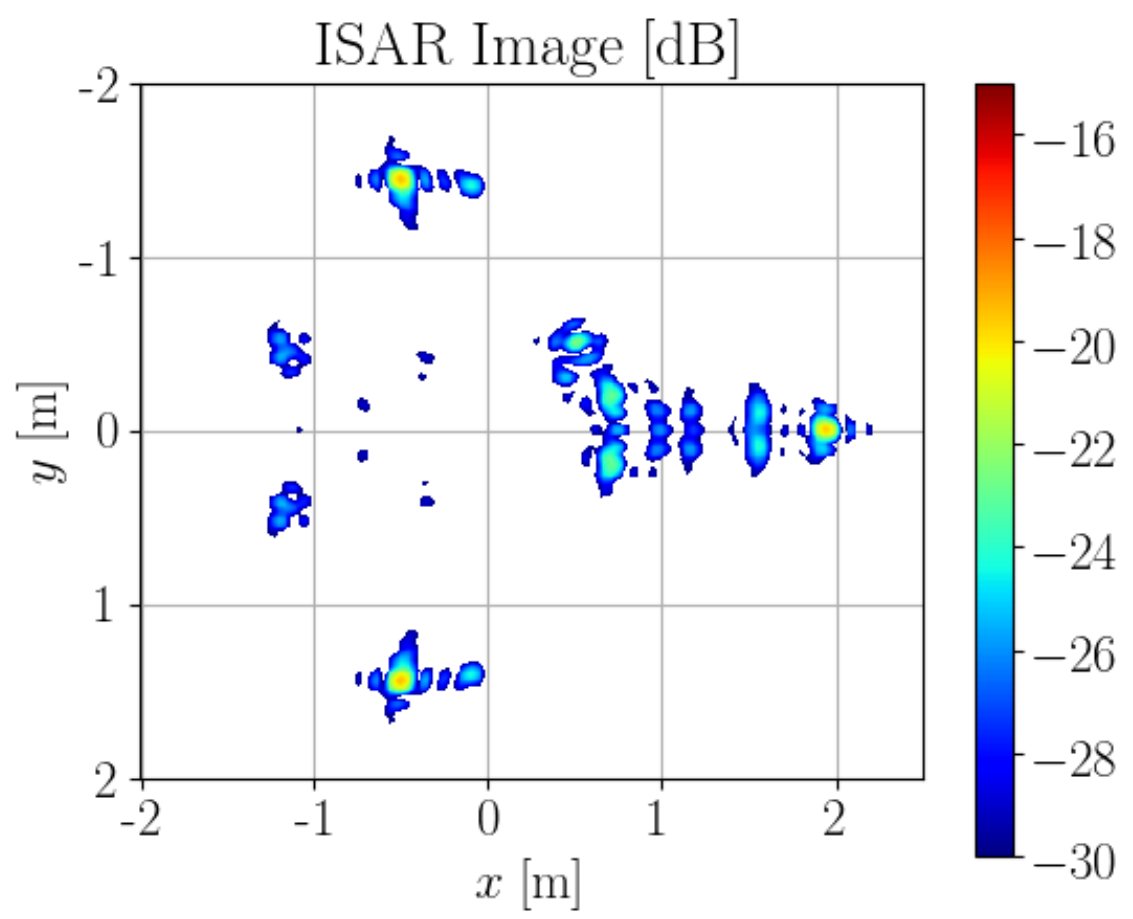

FIGURE 9. ISAR image - HH polarisation.

[14] L. Medgyesi-Mitschang And J. Putnam, Electromagnetic scattering from axially inhomogeneous bodies of revolution, IEEE Transactions on Antennas and Propagation, 32(8):797-806, 1984.

[15] J.-C. Nedelec, Acoustic and Electromagnetic Equations, Integral Representations for Harmonic Problems, Springer, 2001.

[16] V. Rokhlin, Diagonal form of translation operators for the Helmholtz equation in three dimensions, Appl. Comput. Harmon. Anal., 1 (1993), 82-93.

[17] J. Simon Extension des méthodes multipoles rapides: résolution pour des seconds membres multiples et applications aux objets diélectriques, Thèse de l'université de Versailles Saint-Quentin-en-Yvelines, 2003.

[18] P. SoudAIS, Iterative solution of a $3 D$ scattering problem from arbitrary shaped multi-dielectric and multi-conducting bodies, IEEE Transactions on Antennas and Propagation, 954-959, 1994.

[19] See http://www.cims.nyu.edu/cmcl/nufft/nufft.html

[20] See http://www.cims.nyu.edu/cmcl/fmm3dlib/fmm3dlib.html

[21] Matlab, version 8.1.0 (R2013a), The MathWorks Inc., Natick, Massachusetts. http://www.mathworks.com.

[22] N. Zerbib, Méthodes de sous-structuration et de décomposition de domaine pour la résolution des équations de Maxwell. Application au rayonnement d'antenne dans un environnement complexe, PhD thesis, universit de Toulouse, 2006. 


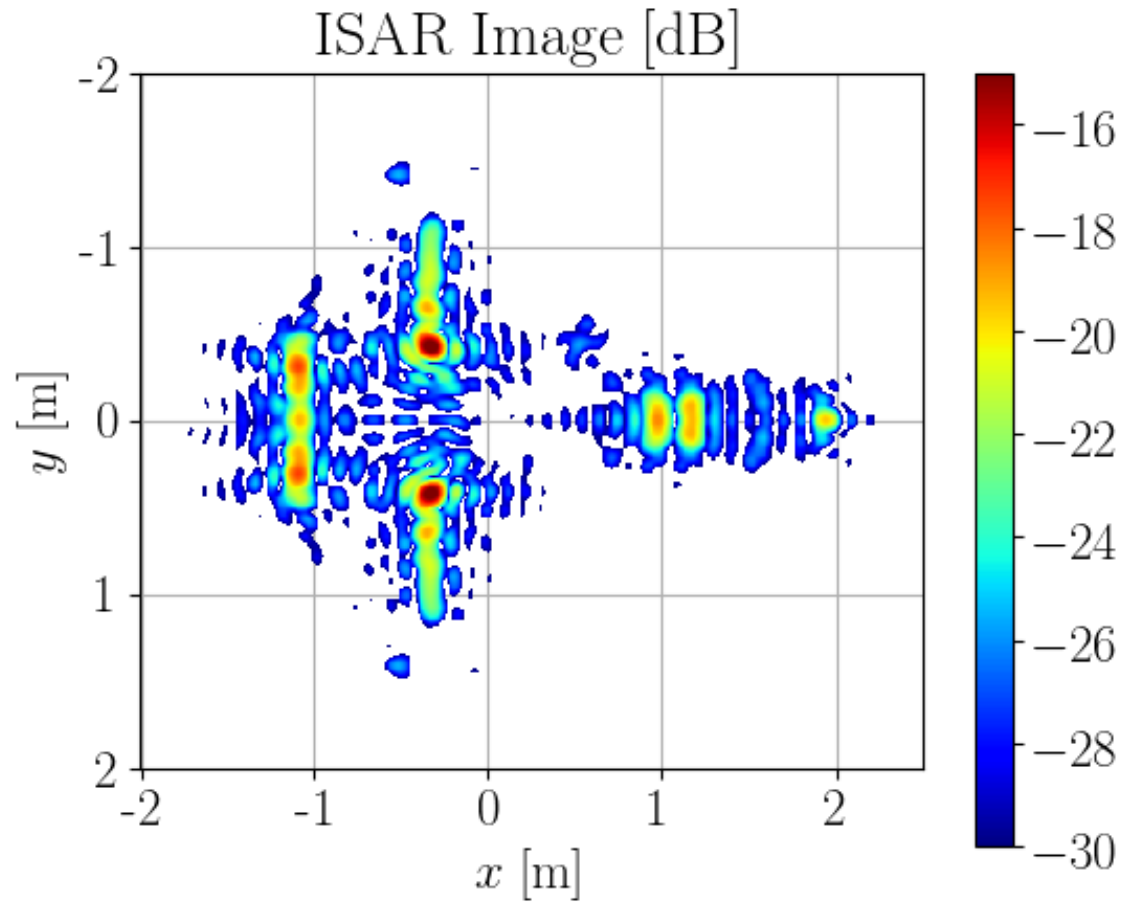

FigURE 10. ISAR image - VV polarisation. 